\title{
A New Motorcycle Simulator Platform: Mechatronics Design, Dynamics Modeling and Control ${ }^{\star}$
}

\author{
L. Nehaoua ${ }^{*}$ S. Hima ${ }^{* *}$ H. Arioui ${ }^{* *}$ N. Séguy ${ }^{* *}$ \\ * INRETS, 2 Av Gl Malleret-Joinville, 94114, Arceuil, France (e-mail: \\ nehaoua@inrets.fr). \\ ** IBISC LSC-LAMI CNRS-FRE 2873, 40 rue Pelvoux 91020, Evry, \\ France(e-mail: arioui,hima,seguy@iup.univ-evry.fr).
}

\begin{abstract}
:
This paper presents the various stages for the construction of a two wheeled riding simulator. Despite its simplicity, the particularity of this simulator comes from the possibility to reproduce most of the movements and the inertial effects allowing to perceive sensations close to reality cases. This simulator has been developed for three purposes:

- as a training tool for new riders in normal traffic environment

- sensibilization for dangerous riding situations (avoidance, emergency braking, failling, etc.)

- to study riders behaviours in such situations

Our studies have lead to an original 5 degrees of freedom (DOF) mechanical platform including a double haptic feedback on the handlebar. The choices of the platform movements and the system actuation are motivated and described.

Also, a detailed kinematics and dynamics modeling will be presented. Some results are shown, validating the actutation requirements and platform control.
\end{abstract}

\section{INTRODUCTION}

Road safety has become a major political and economical issue. While all matters were invested to improve the comfort and safety of drivers cars, those of two-wheeled vehicles remained of last priority. In recent years, public awareness and ecological challenge forced the government to react. After the blow-up of fuel prices and traffic congestion, especially in big agglomeration, the conversion to the use of two-wheeled vehicles know a continuing growth. For all this reasons, the research institutions endeavour as soon as possible to bring adapted answers to the problem of motorcyclists safety by developing works on riders behaviour, whose risk of fatal accident is much higher compared to car drivers.

Driving simulators were used extensively in aeronautical and automotive fields. It remains a secure, low cost and ecological tool for training future drivers and developping new technological features. The main theories used in this area are developed for flight simulation. However, adaptation of these techniques to other simulators (cars and motorcycles) is possible but not direct. Indeed, the dynamics of land vehicles is more constrained and humanmachine interaction is more riche. This situation is much more complicated for two-wheeled vehicles, minimization of risk and the lack of visibility leads to fatal consequences, knowing that the power to mass ratio is higher than that in the case of automobile.

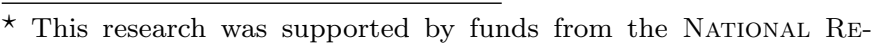
SEARCH AGENCY in SimACOM project.
}

In the past, some motorcycle driving simulators were build. The first prototype was developed by Honda in 1988, with the purpose of studying the motorcycle stability and manoeuvrability Miyamaru et al. [2002]. After 1990, Honda launches out a second prototype of simple simulators with three DOF (roll, pitch and steering), to allow the safe trainee to experience hazardous situations after various traffic conditions on the real city roads. A third prototype was developed using a six DOF parallel manipulator to plan the motion of the platform, and a head mounted display (HMD) for visual projection Chiyoda et al. [2000].

IN 1995, the MORIS simulator project was started by the PERCRO Laboratory, with the aim of developing a tool for designers to acquire knowledge on motorcycle handling and stability as well as the rider control behavior implications on motorcycle performances Ferrazzin et al. [2003]. It consists of a real scooter mock-up mounted on a Stewart parallel platform with seven DOF (include steering axis).

Finally, a motorcycle simulator prototype has been designed at Mechanical Engineering Department at the University of Padova in order to study the man-vehicle interaction in safe conditions Cossalter et al. [2004]. This interaction allows to develop a rider control model. The mechanical platform is a simple structure with five DOF (lateral, roll, pitch, yaw and steering) actuated by five electric servomotors.

\section{PRELIMINARIES}

The choice of the simulator architecture is guided by the necessary needs to have a sufficient perception during the 
riding simulation. Our goal is to reproduce the important inertial effects perceived for the application needs but not all the motorcycle movements. So, the real amplitude of the various DOF was not a dominating object during the design phase.

From these considerations, the number of DOF privileged for our architecture is determined by the simulator's application. We aim to conceive a mechanical platform for the training and the behavioral study of the two wheels vehicle users. After several investigations, three rotations were privileged:

- roll: for the reproduction of short cornering (slalom, way changes),

- pitch: to restitute the accelerations movements illusion as well as fork movements,

- yaw: to reproduce the rear motorcycle wheel skid like in close accident situations. The skid of the front wheel is not reproduced because it is immediately fatal. Indeed, the time between the stable and instable states is extremely short in this case. So, it is difficult to feel it in a real situation and thus it is useless to restitute it to learn any correction technics which is impossible in reality.

Otherwise, the multiplication of the perception stimuli strongly increases the riding simulation sensations Neimer et al. [2005]. Based on this idea, a double haptic feedback is implemented on the handlebar. The first one enables to restitute an inertial delay on the rider bust during the acceleration and braking phases. An effort is created on the motorcyclist arms while varying the distance between the saddle and the handlebar. The second force feedback have the aim to restitute the torque resulting from the tire - road contact, gyroscopic effects and of the front wheel trail Mohellebi et al. [2004].

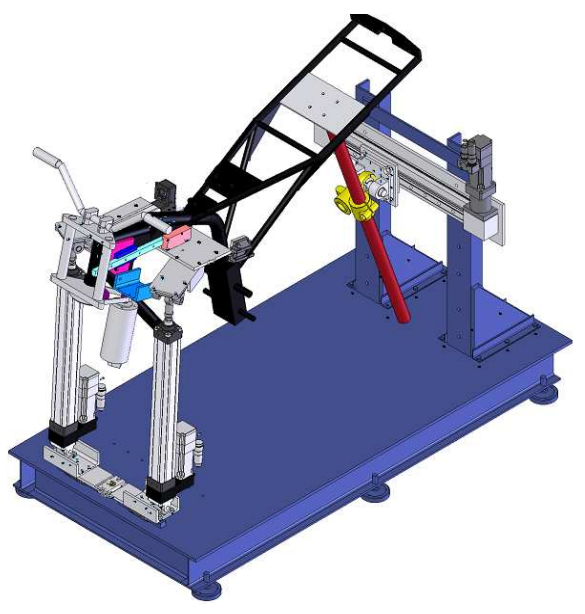

Fig. 1. CAD model of the simulator platform

The position of the various rotation axes is of great importance. No psychophysical study was carried out in the literature except some very simple cases Yamasaki et al. [1998]. Therefore, these axes are taken starting from the real motorcycle kinematics Cossalter [2002]. In order to produce the necessary yaw to feel the rear wheel skid, a slide is positioned on the back of the motorcycle frame. The roll axis is taken in the motorcycle symmetry plane with an ajustable height in order to test several configurations and to find the best perception results. Lastly, for the pitch axis, it is the deplacement of the front fork in the acceleration and braking phases which was privileged, therefore the axis passes by the back of the motorcycle frame. Figure 1 represents the CAD model of the platform simulator which will be described in the next sections.

\section{THE SIMULATOR MECHANICS DESCRIPTION}

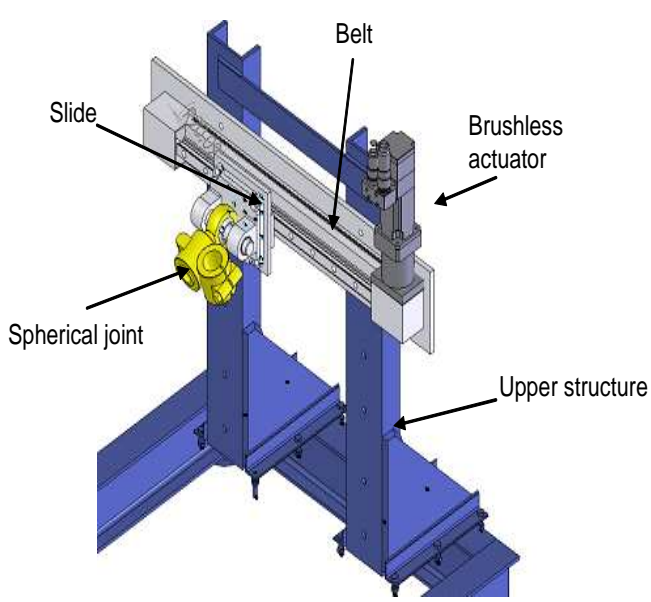

Fig. 2. Upper and lower metallic frames and the yaw rear slide

The simulator platform is composed by an upper and a lower metallic frames. The first consists at two metal structures. An horizontal one, on which all the components of the platform are assembled. The other is vertical mounted at the back of the horizontal structure on which is fixed the slide drive system of the yaw motion (figure 2). The upper platform presents the motorcycle chassis frame.

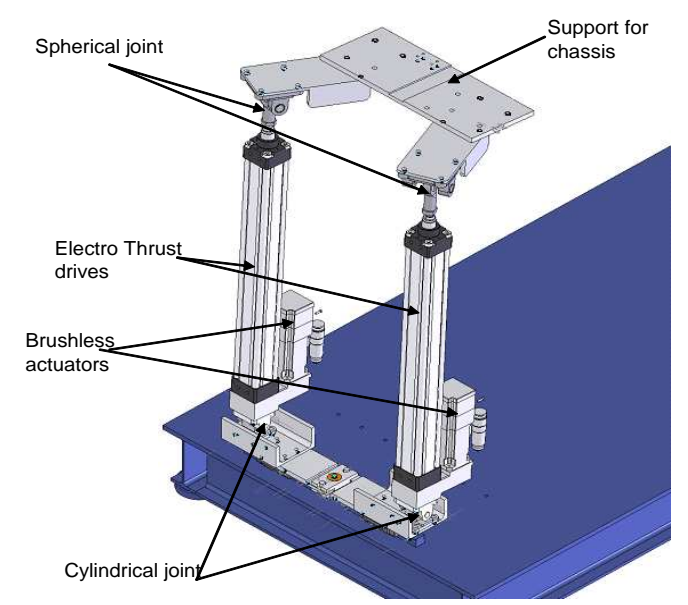

Fig. 3. The two front legs for pitch and roll motion

Two legs are installed at the front of the platform frame (figure 3) to reproduce a rolling and pitching motion of the motorcycle chassis frame. They consist of two ElectroThrust incorporating a high quality ball screw drive. Each leg is connected on the one hand to the lower frame of the simulator by a cylindric joint, and on the other hand 
to the motorcycle chassis by a spherical joint. The two Electro-Thrust are driven respectively with a brushless type servomotor.

For the yaw motion, a slide was fixed on the vertical structure and is driven by a belt actuated by a brushless servomotor via a reductor. A steel bar is fixed rigidly at the rear of the motorcycle chassis frame on one hand and to the rear slide spherical joint on the other hand, thus allowing a yaw motion.

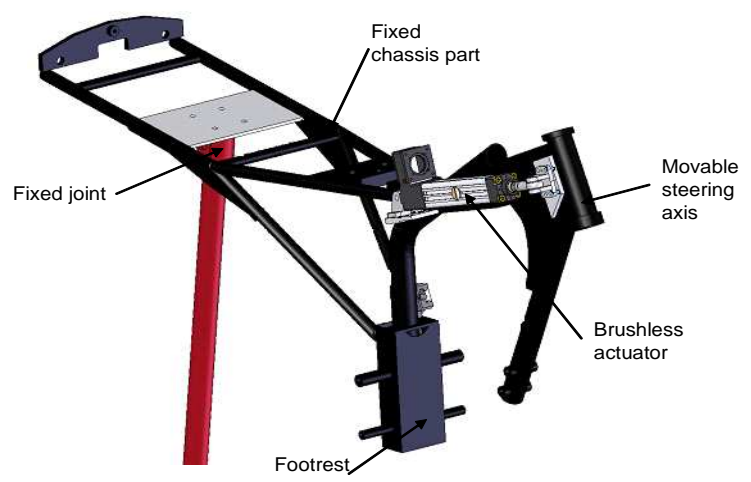

Fig. 4. The modified motorcycle chassis frame

The upper mechanic frame of the mecanical platform presents the motorcycle chassis (figure 4). It is a modified version of a real Yamaha YBR $125 \mathrm{~cm}^{3}$ mock-up. Three supports are designed in order to assemble this mockup to the two front legs and the rear slide bar. The original wheels and suspensions are removed to reduce the mass and inertia of the mock-up. The main motorcycle commands (throttle, brake, clutch levers and the the gearbox selector) have been instrumented, which allow the acquisition of the rider actions. The original mock-up dashboard is used in order to get a visual feedback of the motorcycle speed, engine mode and all other signalling.

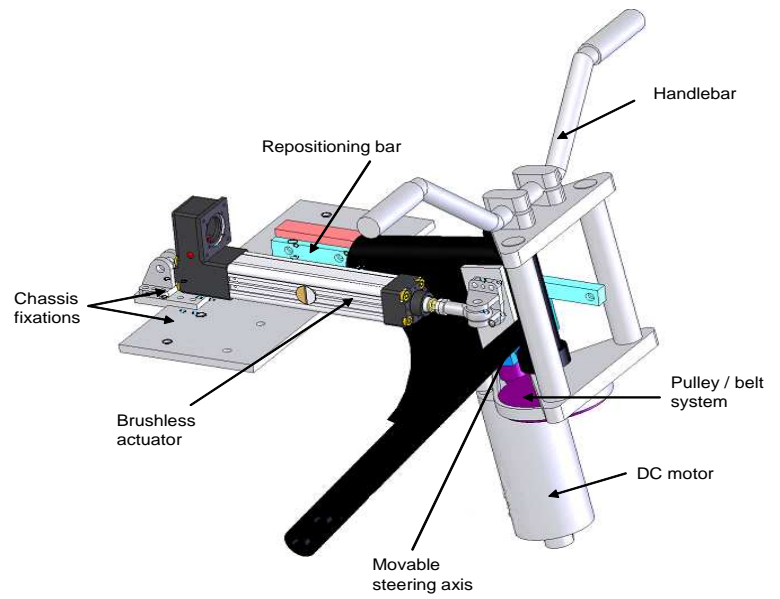

Fig. 5. The double haptic feedback system of the handelbar

The steering axis of the motorcycle handlebar was separated from the motocycle chassis frame. Both are connected by an Electro-Thrust leg driven by a Brushless servomotor to allow small displacements of the handlebar to vary its position with respect to the motorcycle saddle and thus, to simulate an inertial effect exerted on the rider arms during acceleration and braking phases. In addition, the steering rotation axis is driven by a DC motor which permits to simulate on the handelbar an haptic feedback, resulting from the tire/road contact (figure 5).

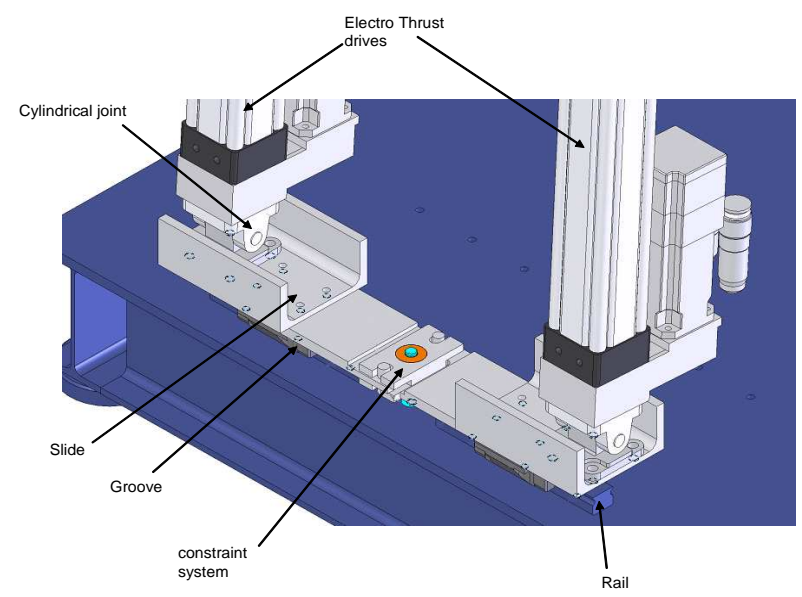

Fig. 6. The symmetric constraint for the $O_{1}$ and $O_{2}$ pivot joints

Finally, figure 6 shows the mechanical system that impose a symmetric displacement constraint of the two legs, and thus, preserving the roll rotation axis in the vertical motorcycle symmetric plane.

\section{INVERSE KINEMATICS FORMULAION}

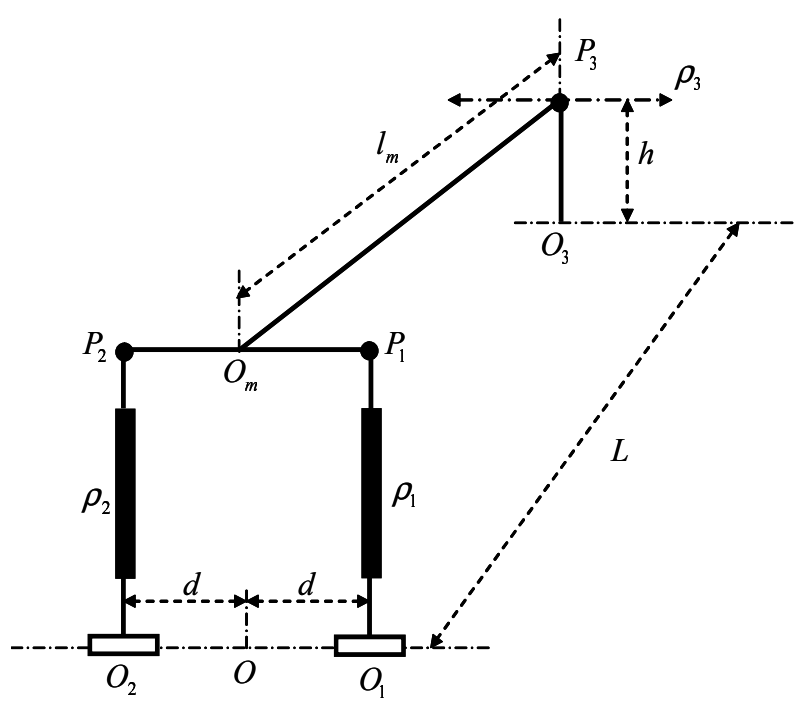

Fig. 7. Kinematics scheme of the simulator's platform

Let $\Re(O, i, j, k)$ to be the fixed reference and $\Re_{m}\left(O_{m}, i_{m}, j_{m}, k_{m}\right)$ the platform's mobile reference. $O_{1}, O_{2}$ and $O_{3}$ are repectively the attachment points of the two legs and the rear slide with the simulator's base. $P_{1}, P_{2}$ and $P_{3}$ are repectively the attachment points of the two legs and the rear slide with the upper mobile platform. The configuration of the reference frame $\Re_{m}$ is characterized by the position $\left(x_{m}, y_{m}, z_{m}\right)$ of its origin and three Euler orientation angles $(\psi, \theta, \varphi)$, corresponding respectively to yaw, pitch 
and roll. Taking the Z-Y-X convention, rotation matrix is computed as following:

$$
R=R_{\psi} R_{\theta} R_{\varphi}=\left(\begin{array}{lll}
r_{11} & r_{12} & r_{13} \\
r_{21} & r_{22} & r_{23} \\
r_{31} & r_{32} & r_{33}
\end{array}\right)
$$

or in a detailed form, where $c \equiv \cos$ and $s \equiv \sin$ :

$$
\mathbf{R}=\left(\begin{array}{ccc}
c \theta c \psi & s \varphi s \theta c \psi-c \varphi s \psi & c \varphi s \theta c \psi+s \varphi s \psi \\
c \theta s \psi & s \varphi s \theta s \psi+c \varphi c \psi & c \varphi s \theta s \psi-s \varphi c \psi \\
-s \theta & s \varphi c \theta & c \varphi c \theta
\end{array}\right)
$$

Vector $\mathbf{O P}_{\mathbf{3}}$ is given in the fixed base reference by $\mathbf{O} \mathbf{P}_{\mathbf{3}}{ }^{O}=$ $\left(-L, \rho_{3}, h\right)^{T}$. Using transformation matrix $\mathbf{R}$ the same vector can be written such as:

$$
\mathbf{O P}_{\mathbf{3}}{ }^{O}=\mathbf{O O}_{\mathbf{m}}{ }^{O}+\mathbf{R O}_{\mathbf{m}} \mathbf{P}_{\mathbf{3}}{ }^{m}
$$

where, $\mathbf{O} \mathbf{O}_{\mathbf{m}}{ }^{O}=\left(x_{m}, y_{m}, z_{m}\right)^{T}$ and $\mathbf{O}_{\mathbf{m}} \mathbf{P}_{\mathbf{3}}{ }^{m}=\left(-l_{m}, 0,0\right)^{T}$. Replacing the different vectors components in equation (3) we can deduce the coordinates of the mobile reference origin $O_{m}$ and the rear slide displacement $\rho_{3}$ as following:

$$
\left\{\begin{array}{l}
x_{m}=-L+l_{m} r_{11} \\
y_{m}=0 \\
z_{m}=h-l_{m} r_{31} \\
\rho_{3}=-l_{m} r_{21}
\end{array}\right.
$$

where $L, h$ and $l_{m}$ are geometric constants (figure 7).

Now, the leg vector equation for $i=1,2$ is given by:

$$
\mathbf{O}_{\mathbf{i}} \mathbf{P}_{\mathbf{i}}{ }^{O}=\mathbf{O}_{\mathbf{i}} \mathbf{O}^{O}+\mathbf{O O}_{\mathbf{m}}{ }^{O}+\mathbf{R O}_{\mathbf{m}} \mathbf{P}_{\mathbf{i}}{ }^{m}
$$

where, $\mathbf{O}_{\mathbf{m}} \mathbf{P}_{\mathbf{i}}{ }^{m}=l\left(0,(-1)^{i+1}, 0\right)^{T}, \mathbf{O}_{\mathbf{i}} \mathbf{O}^{O}=(-1)^{i} d \mathbf{j}$ and $d$ is the coordinate of the two cylindrical joints $O_{1}$ and $\mathrm{O}_{2} . l$ is the distance between $P_{1}$ and $P_{2}$. Substituting in equation (5), the components of vectors $\mathbf{O}_{\mathbf{i}} \mathbf{P}_{\mathbf{i}}{ }^{O}=\rho_{i} u_{i}$ can be deduced as following:

$$
\rho_{i} \mathbf{u}_{\mathbf{i}}=\left(\begin{array}{c}
-L+l_{m} r_{11}+(-1)^{i+1} l r_{12} \\
0 \\
h+l_{m} r_{31}+(-1)^{i+1} l r_{32}
\end{array}\right)
$$

and

$$
d=l r_{22}
$$

where, $\mathbf{u}_{\mathbf{i}}$ is the unit vector along the leg axis, $\mathbf{j}=(0,1,0)^{T}$ and $\rho_{i}^{2}=\mathbf{O}_{\mathbf{i}} \mathbf{P}_{\mathbf{i}}^{T} \mathbf{O}_{\mathbf{i}} \mathbf{P}_{\mathbf{i}}$ are the legs lengths.

To determine the inverse Jacobian matrix, we note the Euler angle rates vector by $\dot{\mathbf{q}}_{\mathbf{r}}=(\dot{\psi}, \dot{\theta}, \dot{\varphi})^{T}$. The velocity of the leg elongation is given by:

$$
\dot{\rho}_{i}=\mathbf{O}_{\mathbf{i}} \dot{\mathbf{P}}_{\mathbf{i}}^{T} \mathbf{u}_{\mathbf{i}}
$$

Deriving equation (5) and replacing in (8) we find that:

$$
\dot{\rho}_{i}=(-1)^{i} \mathbf{u}_{\mathbf{i}}^{T} \dot{d} \mathbf{j}+\mathbf{u}_{\mathbf{i}}^{T} . \mathbf{O} \dot{\mathbf{O}}_{\mathbf{m}}+\left(\omega \times \mathbf{O}_{\mathbf{m}} \mathbf{P}_{\mathbf{i}}\right)^{T} \mathbf{u}_{\mathbf{i}}
$$

where $\omega=\gamma \dot{\mathbf{q}}_{\mathrm{r}}$ is the mobile platform angular velocity expressed in the fixed reference frame, and $\gamma$ is the matrix transformation between the angular velocity and Euler angles rates. Equation (9) can be written using the mixed vector product property $(\mathbf{u} \times \mathbf{v}) \cdot \mathbf{w}=(\mathbf{w} \times \mathbf{u}) \cdot \mathbf{v}$ as following:

$$
\dot{\rho}_{i}=(-1)^{i} \mathbf{u}_{\mathbf{i}}^{T} \dot{d} \mathbf{j}+\mathbf{u}_{\mathbf{i}}^{T} . \mathbf{O} \dot{\mathbf{O}}_{\mathbf{m}}+\left(\mathbf{u}_{\mathbf{i}} \times \mathbf{P}_{\mathbf{i}} \mathbf{O}_{\mathbf{m}}\right)^{T} \omega
$$

For the rear slide velocity, we have $\rho_{3}=\mathbf{j}^{T} \mathbf{O} \dot{\mathbf{P}}_{\mathbf{3}}$. By deriving and rearranging, we get:

$$
\dot{\rho}_{3}=\mathbf{j}^{T} \mathbf{O} \dot{\mathbf{O}}_{\mathbf{m}}+\left(\mathbf{j} \times \mathbf{P}_{\mathbf{3}} \mathbf{O}_{\mathbf{m}}\right)^{T} \omega
$$

From equations (10) and (11) and knowing that $\mathbf{u}_{\mathbf{i}}^{T} \mathbf{j}=0$, we can deduce that:

$$
\dot{\rho}=J_{-1} W
$$

where, $\dot{\rho}=\left(\dot{\rho}_{1}, \dot{\rho}_{2}, \dot{\rho}_{3}\right)^{T}, W=\left(\mathbf{O} \dot{\mathbf{O}}_{\mathbf{m}}{ }^{T}, \omega^{T}\right)$ and the inverse Jacobian matrix is:

$$
J_{-1}=\left[\begin{array}{cc}
\mathbf{u}_{\mathbf{1}}^{T} & \left(\mathbf{u}_{\mathbf{1}} \times \mathbf{P}_{\mathbf{1}} \mathbf{O}_{\mathbf{m}}\right)^{T} \\
\mathbf{u}_{\mathbf{2}}^{T} & \left(\mathbf{u}_{\mathbf{2}} \times \mathbf{P}_{\mathbf{2}} \mathbf{O}_{\mathbf{m}}\right)^{T} \\
\mathbf{j}^{T} & \left(\mathbf{j} \times \mathbf{P}_{\mathbf{3}} \mathbf{O}_{\mathbf{m}}\right)^{T}
\end{array}\right]
$$

The platform is designed to perform three rotations of maximum $\pm 15^{\circ}$, The inverse Jacobian matrix is always invertible within this intervall, so there is no singularity of the platform within its workspace.

\section{DYNAMICS OF THE PLATFORM}

In this section, we look to develop a dynamic model of the mechanical platform to characterize the simulator's capabilities. Applying Newton principal on the mobile platform gives (Dasgupta and Mruthyunjaya [1998]):

$$
m_{p} \mathbf{g}+\sum_{i=1}^{2} \mathbf{F}_{\mathbf{u i}}+\sum_{i=1}^{2} \mathbf{F}_{\mathbf{n i}}+\mathbf{F}_{\mathbf{3}}=m_{p} \ddot{\mathbf{O G}}_{p}
$$

where $\mathbf{F}_{\mathbf{3}}=f_{3} \mathbf{j}$ is the reaction force on the rear spherical joint with $f_{3}$ is the motor actuation. The Euler equation of the platform is then written to express the rotational dynamics with respect to the mobile reference frame $\Re_{m}$, so:

$$
\begin{aligned}
& m_{p} \mathbf{O}_{\mathbf{m}} \mathbf{G}_{\mathbf{p}} \times \mathbf{g}+\sum_{i=1}^{2} \mathbf{O}_{\mathbf{m}} \mathbf{P}_{\mathbf{i}} \times \mathbf{F}_{\mathbf{u i}}+\sum_{i=1}^{2} \mathbf{O}_{\mathbf{m}} \mathbf{P}_{\mathbf{i}} \times \mathbf{F}_{\mathbf{n i}}+ \\
& \mathbf{O}_{\mathbf{m}} \mathbf{P}_{\mathbf{3}} \times \mathbf{F}_{\mathbf{3}}=m_{p} \mathbf{O}_{\mathbf{m}} \mathbf{G}_{\mathbf{p}} \times \mathbf{O}^{\mathbf{G}_{p}}+I_{p} \dot{\omega}+\omega \times I_{p} \omega
\end{aligned}
$$

where $\mathbf{O}_{\mathbf{m}} \mathbf{G}_{\mathbf{p}}$ is the position of the mobile platform center of gravity $G_{p}$ with respect to point $O_{m}$ expressed in the base reference. $I_{p}, m_{p}$ are respectively the platform inertia tensor and mass. $\omega$ is the rotational velocity. The platform center of gravity acceleration is given by the following equations:

$$
\mathbf{O}_{\mathbf{m}} \ddot{G}_{\mathbf{p}}=\mathbf{O} \ddot{\mathbf{O}}_{\mathbf{m}}+\dot{\omega} \times \mathbf{O}_{\mathbf{m}} \mathbf{G}_{\mathbf{p}}+\omega \times\left(\omega \times \mathbf{O}_{\mathbf{m}} \mathbf{G}_{\mathbf{p}}\right)
$$

Combining Newton and Euler equations (14) and (15) into one formula and replacing $\mathbf{O}_{\mathbf{m}} \mathbf{G}_{\mathbf{p}}$ by its expression, we obtain the dynamics equation of the platform as:

$$
\mathbf{M} \dot{\mathbf{W}}+\mathbf{C}_{\omega}+\mathbf{G}_{g}=J_{-1}^{T} \mathbf{F}
$$

where $J_{-1}^{T}$ is the transpose of the inverse jacobian matrix established in the inverse kinematics section. $\mathbf{M}$ is the mass matrix, $\mathbf{C}_{\omega}$ vector including the nonlinear terms and function of $\omega$ and $\mathbf{G}_{g}$ the gravity term.

However, the simulator platform has only three DOF corresponding to Euler rotations, then $\mathbf{O O}_{\mathbf{m}}=\Gamma \dot{\mathbf{q}}_{\mathbf{r}}$ and $\omega=\gamma \mathbf{q}_{\mathbf{r}}$, so it is well convenient to express the dynamic formulation in function of $\dot{\mathbf{q}}_{\mathbf{r}}$ rather then $\mathbf{W}$. Introducing 
Lagrange multipliers $\lambda$, the dynamics of the simulator's platform is given by:

$$
\mathbf{M} \Phi \ddot{\mathbf{q}}_{\mathbf{r}}+M \dot{\Phi} \dot{\mathbf{q}}_{\mathbf{r}}+\mathbf{C}_{\omega}+\mathbf{G}_{g}=\left[\begin{array}{ll}
J_{-1}^{T}-C_{q}^{T}
\end{array}\right]\left[\begin{array}{c}
F \\
\lambda
\end{array}\right]
$$

$\Phi=\left[\Gamma^{T} \gamma^{T}\right]^{T}$ and $C_{q}^{T}$ is transpose of the Jacobian matrix of the constraint equation (4).

\section{EXPERIMENTATION}

An experiment is led on the simulator's platform (figure 8) to show the advantage of using a dynamic model to conceive a trajectory tracking controller.

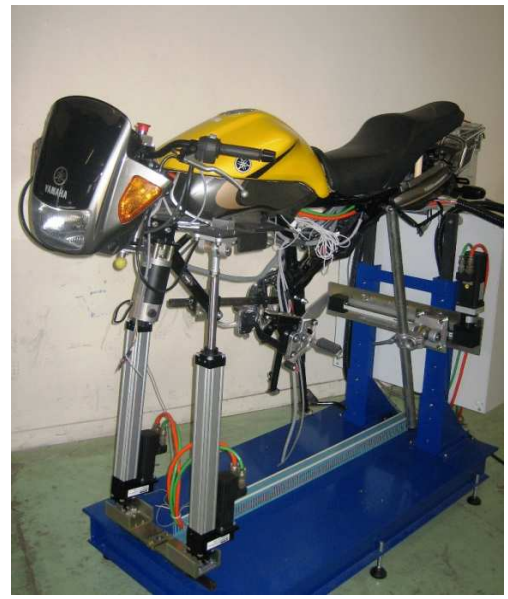

Fig. 8. Constructed riding motorcycle simulator

We choose to explore just the platform's pitch degree of freedom . A driver on the simulator handles the gas throttle and the brake lever to accelerate and slow down. Figure 10 shows the recorded acceleration profile, as well as the corresponding pitch angle calculated by the motion cueing model (for more information on the general simulation scheme and the motion cueing algorithm refer to Nehaoua et al. [2007], Nehaoua et al. [2006]).

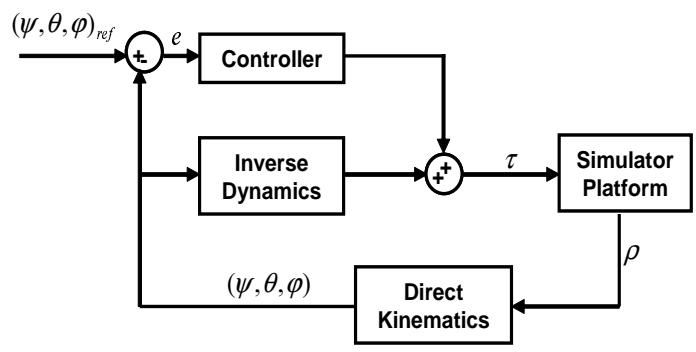

Fig. 9. Control scheme used to drive the simulator's platform

A simple Controller is implemented based on a ProportionnalDerivative to track the reference trajectory and a gravity compensation bloc (figure 9 ). The control law is given by:

$$
\begin{aligned}
& F=-J_{-1}^{-T}\left(\mathbf{M} \dot{\Phi} \dot{\mathbf{q}}_{\mathbf{r}}+\mathbf{C}_{\omega}+\mathbf{G}_{g}\right)+ \\
& J_{-1}^{-T} \mathbf{M} \Phi\left(K_{p}\left(\mathbf{q}_{\mathbf{r e f}}-\mathbf{q}_{r}\right)-K_{d} \dot{\mathbf{q}}_{\mathbf{r}}\right)
\end{aligned}
$$

where, $K_{p}, K_{d}$ are the PD gains and $\mathbf{q}_{\text {ref }}$ is the reference trajectory.
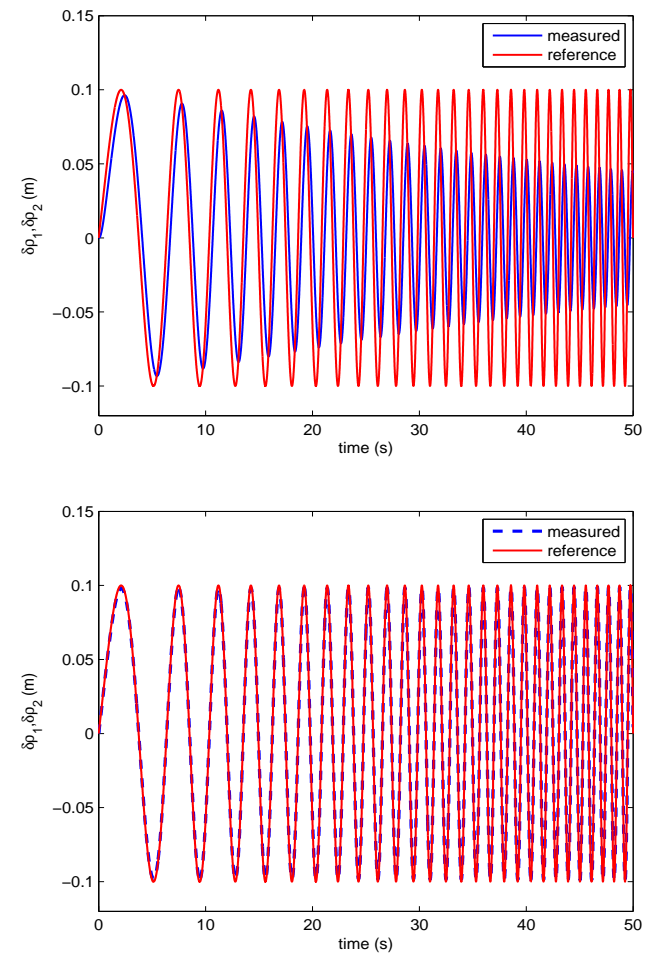

Fig. 10. Measured and reference legs elongation (a)without correction (b)with correction
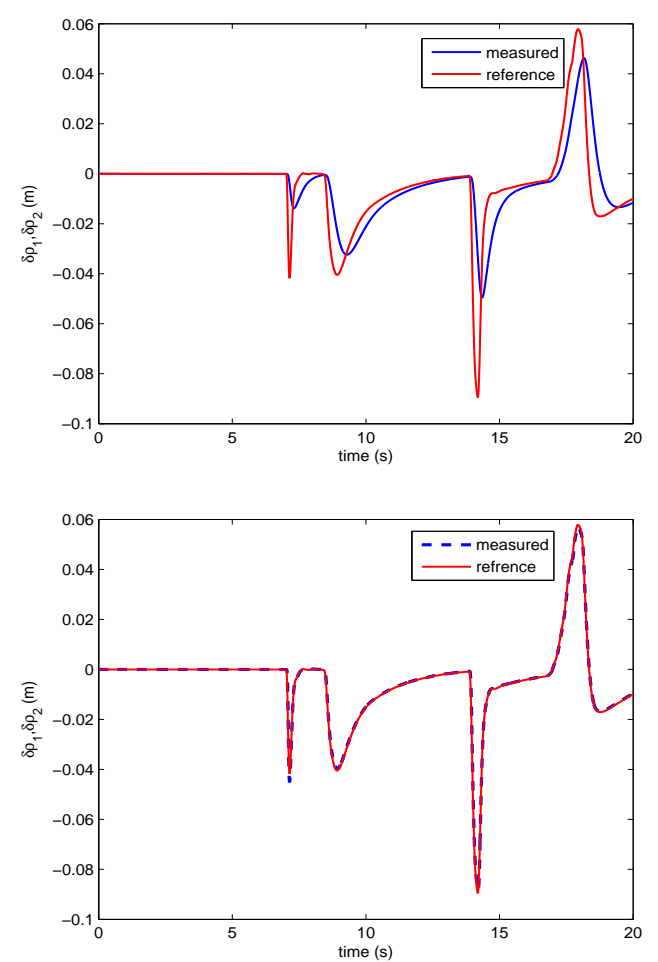

Fig. 11. Reference leg elongation $\rho_{1}, \rho_{2}$ and tracked trajectories using dynamic modeling in an acceleration/braking maoeuver

Figure 11 shows the legs elongations $\rho_{1}$ and $\rho_{2}\left(\rho_{3}=0\right.$ since thre is no yaw motion). The dotted curve corresponds 
to the reference elongations as calculated by the inverse kinematics model. We can see that with the control scheme described by equation (30), we have a best tracking of the reference trajectories, at the price of a more computing time. However, the used acquisition module (a home electronic card based on a V853 microcontroller) is piloted maximally at $1000 \mathrm{~Hz}$, therefore, the use of a linearized dynamic model doesn't pose any realtime constraint.

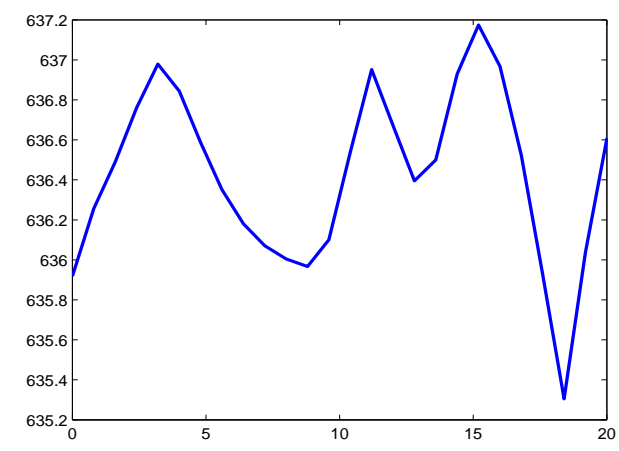

Fig. 12. Input servocontroller forces $f_{1}$ and $f_{2}(N)$

The input forces $f_{1}$ and $f_{2}$ to the servocontroller modeules are shown in figure 12 . Figure 13 represents the corresponding torque developed by the brushless motors given by $\tau_{i}=\frac{p}{2 \pi \eta} f_{i}$, where, $p$ and $\eta$ are respectively the screwnut lead and effeciency. Consequently, the platform is sufficiently actionned to reach the required dynamic for a given driving situation.

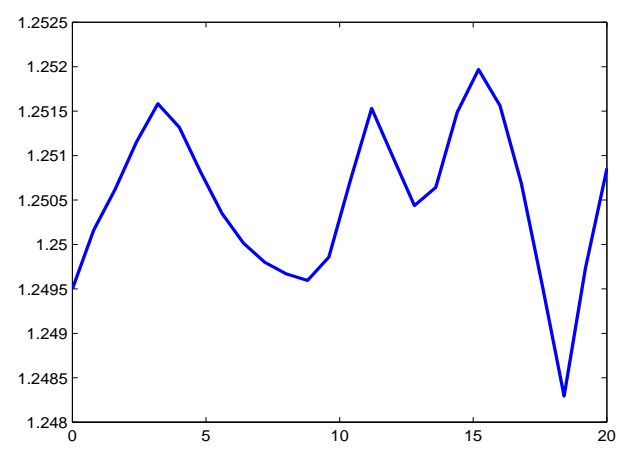

Fig. 13. Developed actuator's torques $N / m$

\section{CONCLUSION}

In the first part of this paper, the important points retained to conceive a two wheels riding simulator have been listed. The application to which it is dedicated and the necessary perception guided us to choose the mechanical architecture.

The mechanical design has been described and CAD model of each part is illustrated. The inverse kinematics of the platform was presented allowing to transform the motion cueing algorithm trajectories into actuator inputs.

Also, a detailed dynamics modeling of the simulator's platform is developed. The validation experiments of per- formances are satisfactory and permits to reach our objectives for normal, dangerous or extreme situations. The use of control law based on the inverse dynamics prove its effectiveness compared to results obtained by using just the inverse kinematics model.

Future work will be concern three principle axes. Firstly, a more detailed dynamic model will be implemented by introducing friction terms. Secondly, an online identification method to get a more accurate platform parameters. Finally, more effort to be devoted on robust controllers.

\section{REFERENCES}

S. Chiyoda, K. Yoshimoto, D. Kawasaki, Y. Murakami, and T. Sugimoto. Development of a motorcycle simulator using parallel manipulator and head mounted display. In Driving Simulation Conference(DSC00), Paris,France, 2000.

V. Cossalter. Motorcycle Dynamics. Race Dynamics Inc, ISBN: 0-9720514-0-6, Milwaukee, USA, 2002.

V. Cossalter, A. Doria, and R. Lot. Development and validation of a motorcycle riding simulator. In World Automotive Congress(FISITA2004), Barcelona, Spain, May 2004.

B. Dasgupta and T.S. Mruthyunjaya. Closed-form dynamic equations of the general stewart platform through the newton-euler approach. Mech. Mach.Theory, 33: 993-1012, 1998.

D. Ferrazzin, F. Barnagli, C.A. Avizzano, G.Di Pietro, and M. Bergamasco. Designing new commercial motorcycles through a highly reconfigurable virtual reality-based simulator. Journal of Advanced Robotics, 17(4):293-318, 2003.

Y. Miyamaru, G. Yamasaki, and K. Aoki. Development of a motorcycle riding simulator. Society of Automotive Engineers of Japan, 23:121-126, 2002.

H. Mohellebi, S. Espié, and A. Kheddar. Adaptive haptic steering wheel for driving simulators. In Proceedings of 2004 IEEE/RSJ International Conference on Robots and Intelligent Systems (IROS04), June 2004.

L. Nehaoua, H. Arioui, H. Mohellebi, and S. Espié. Restitution Movement for a Low Cost Driving Simulator. In Proceedings of the 2006 American Control Conference (ACC06), pages 2599-2604, Minneapolis, Minnesota, June 2006.

L. Nehaoua, S. Hima, H. Arioui, N. Seguy, and S. Espie. Design and modeling of a new motorcycle riding simulator. American Control Conference (ACCO 7 ), pages 171-181, 2007.

J. Neimer, H. Mohellebi, S. Espié, and A. Kheddar. Optimization of linear motion base dedicated to normal driving conditions. In Driving Simulator Conference (DSC05), Orlando, Florida, 30 nov-2 dec 2005.

G. Yamasaki, K. Aoki, Y. Miyamaru, and K. Ohnuma. Development of motorcycle training simulator. Society of Automotive Engineers of Japan, 19:81-85, 1998. 